Voix et Images

volxetimages

\title{
Images de la production scénique des écritures de Daniel Danis
}

\section{Gilbert David}

Volume 40, numéro 1 (118), automne 2014

Daniel Danis

URI : https://id.erudit.org/iderudit/1028018ar

DOI : https://doi.org/10.7202/1028018ar

Aller au sommaire du numéro

Éditeur(s)

Université du Québec à Montréal

ISSN

0318-9201 (imprimé)

1705-933X (numérique)

Découvrir la revue

Citer ce document

David, G. (2014). Images de la production scénique des écritures de Daniel

Danis. Voix et Images, 40(1), 27-35. https://doi.org/10.7202/1028018ar d'utilisation que vous pouvez consulter en ligne.

https://apropos.erudit.org/fr/usagers/politique-dutilisation/ 


\title{
IMAGES DE LA PRODUCTION SCÉNIQUE DES ÉCRITURES DE DANIEL DANIS
}

\author{
$+++$ \\ GILBERT DAVID \\ Université de Montréal
}

La présente section propose huit photographies d'autant de productions scéniques des œuvres de Daniel Danis. Dans l'entretien avec l'auteur qui la précède, il n'a pas été question des approches proposées par divers metteurs en scène de ses textes. Il n'y a là aucun mystère, car un auteur de thêâtre est rarement le mieux placé pour évaluer la pertinence ou non des productions de son œuvre - trop heureux d'avoir été choisi par un producteur. De plus, notre dossier est centré sur l'écriture danisienne et assez marginalement sur ses manifestations en présence d'un public.

Néanmoins, il aurait été regrettable de ne pas faire voir au moins quelques images représentatives du passage nécessaire d'un texte de thêâtre à sa représentation, en sélectionnant (surtout) des créations depuis 1993 jusqu'à nos jours, dans la mesure justement où la dramaturgie danisienne pose un grand nombre de défis aux créateurs théâtraux - de l'acteur au metteur en scène et au scénographe - en raison de son questionnement concernant les conventions dominantes. 


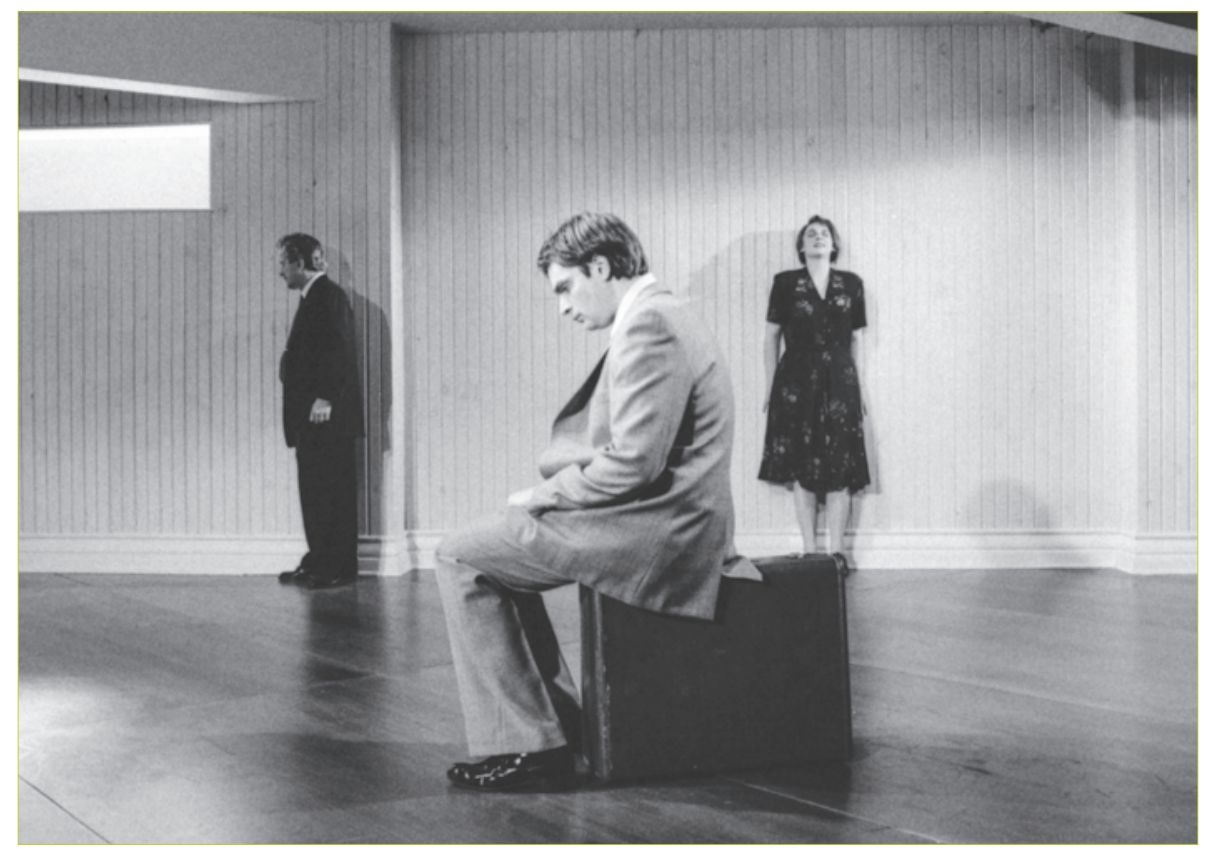

Celle-là de Daniel Danis. Mise en scène de Louise Laprade, production d'ESPACE GO (Montréal), 1993. Avec, de gauche à droite, Marc Legault (Le Vieux), Jean-François Pichette (Le Fils) et Isabelle Miquelon (La Mère). Photo: Yves Renaud. 


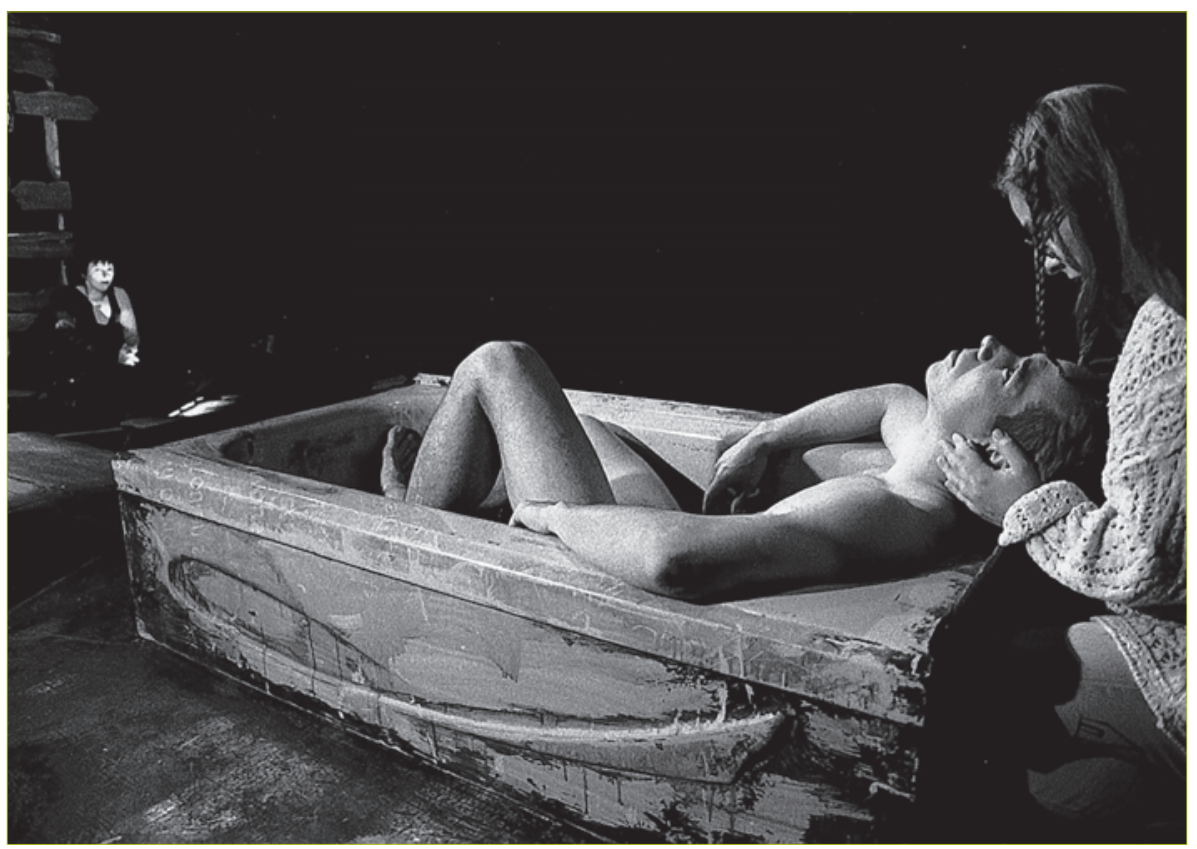

Cendres de cailloux de Daniel Danis. Mise en scène de Gill Champagne, production du Théâtre Blanc (Québec), 1995. Avec, de gauche à droite, Linda Laplante (Shirley), Marco Poulin (Clermont) et Nathalie Poiré (Pascale). Photo: Patrick Fabre. 


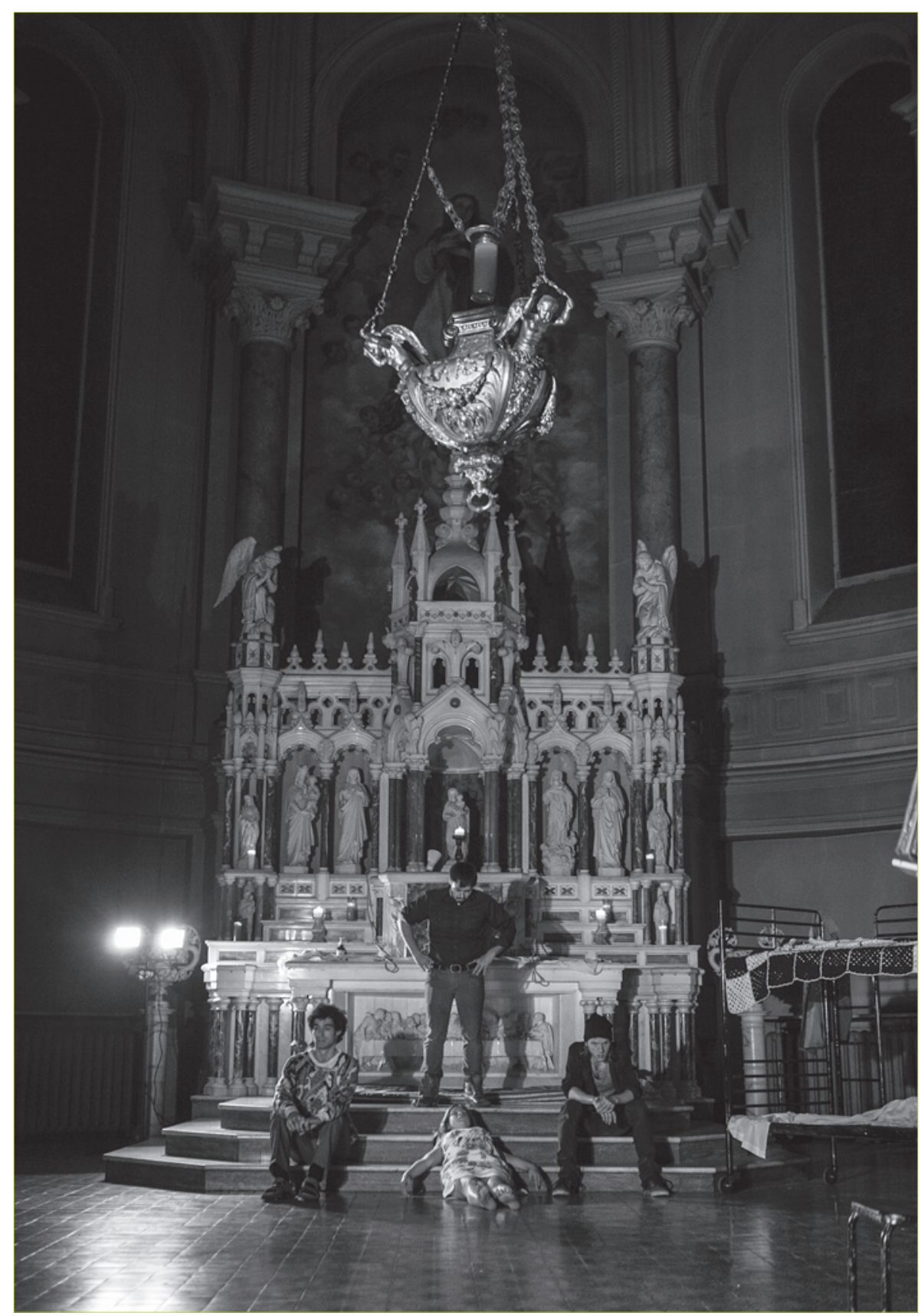

Le chant du Dire-Dire de Daniel Danis. Mise en scène de Marc Béland, production du Théâtre le Mimésis (Montréal), église de l'Immaculée-Conception, 2012. Avec, de gauche à droite, Guillaume Regaudie (Fred-Gilles), Louis-Philippe Tremblay (Rock), Marie-France Bédard (Noéma) et Yves-Antoine Rivest (William). Photo: Hugo B. Lefort. 


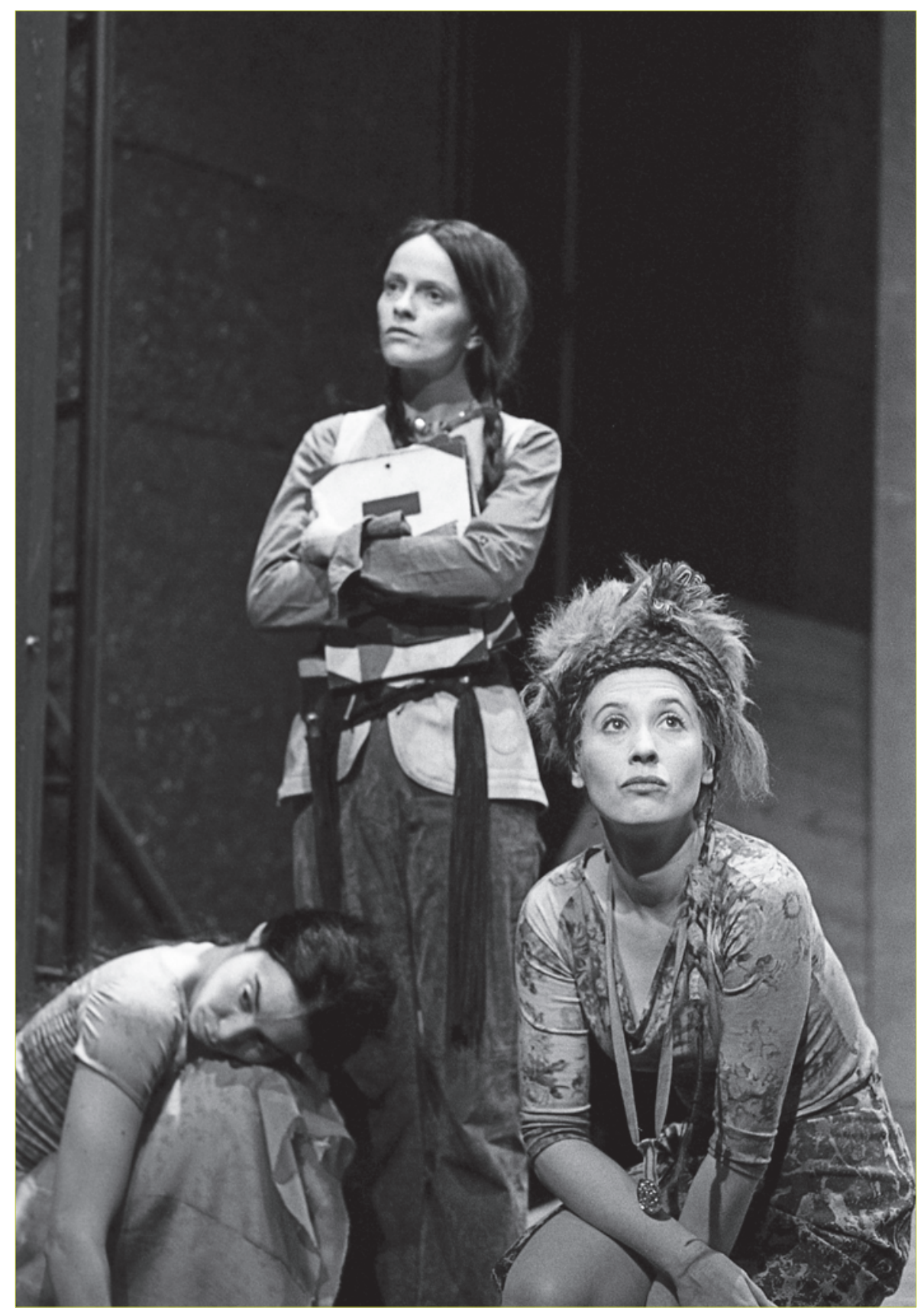

Le langue-à-langue des chiens de roche de Daniel Danis. Mise en scène de Michel Didym, production de la Comédie-Française au Théâtre du Vieux-Colombier (Paris), 2001. Avec, de gauche à droite, Bérangère Allaux (Djoukie), Anne Kessler (Joëlle) et Cécile Brune (Déesse). Photo: Éric Didym. 


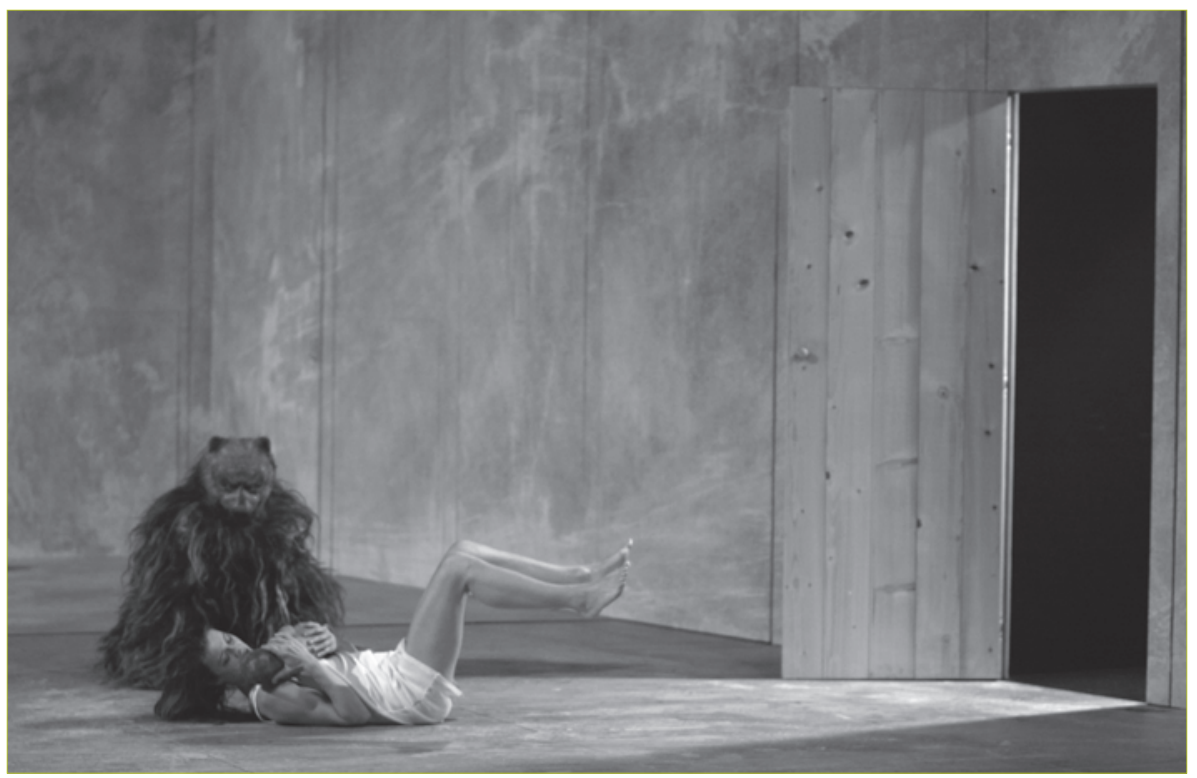

e. Roman-dit de Daniel Danis. Mise en scène d'Alain Françon, production du Théâtre de la Colline (Paris), 2005. Avec, de gauche à droite, Stéphanie Béghain (Noiraude) et Caroline Proust (Romane). Photo: Pascal Victor. 


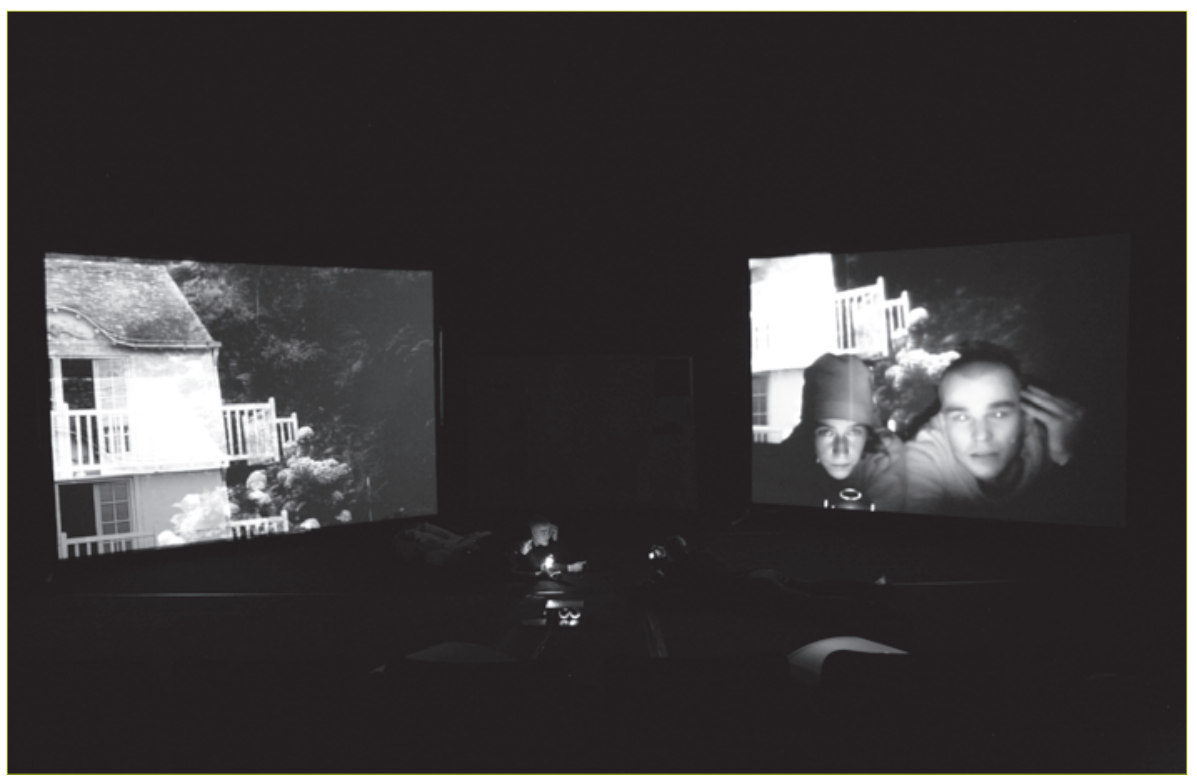

Kiwi de Daniel Danis. Écriture scénique de l'auteur, coproduction Le Grand Bleu (Lille), Compagnie Daniel Danis, arts/sciences (Montréal) et autres. Avec, de gauche à droite, Marie Delhaye (Kiwi) et Baptiste Amann (Litchi). Photo: Krista Boggs. 


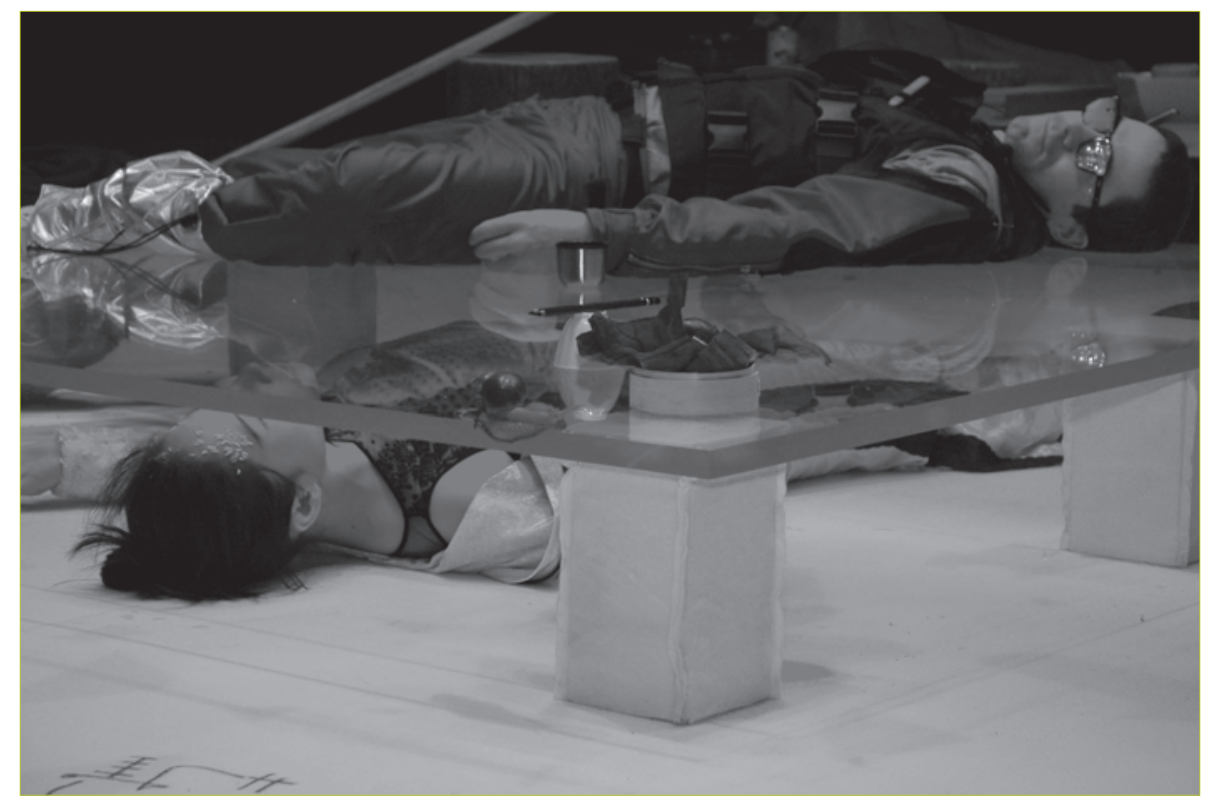

Sommeil et rouge (d'après Chant de l'éternel regret de Po Kui-Yi) de Daniel Danis. Écriture scénique de l'auteur, coproduction de la Compagnie Daniel Danis, arts/sciences (Québec) et autres, Usine C, 2006. Avec, de haut en bas, Daniel Danis et la danseuse Ziyian Kwan. Photo: Jasmin Le Blanc. 


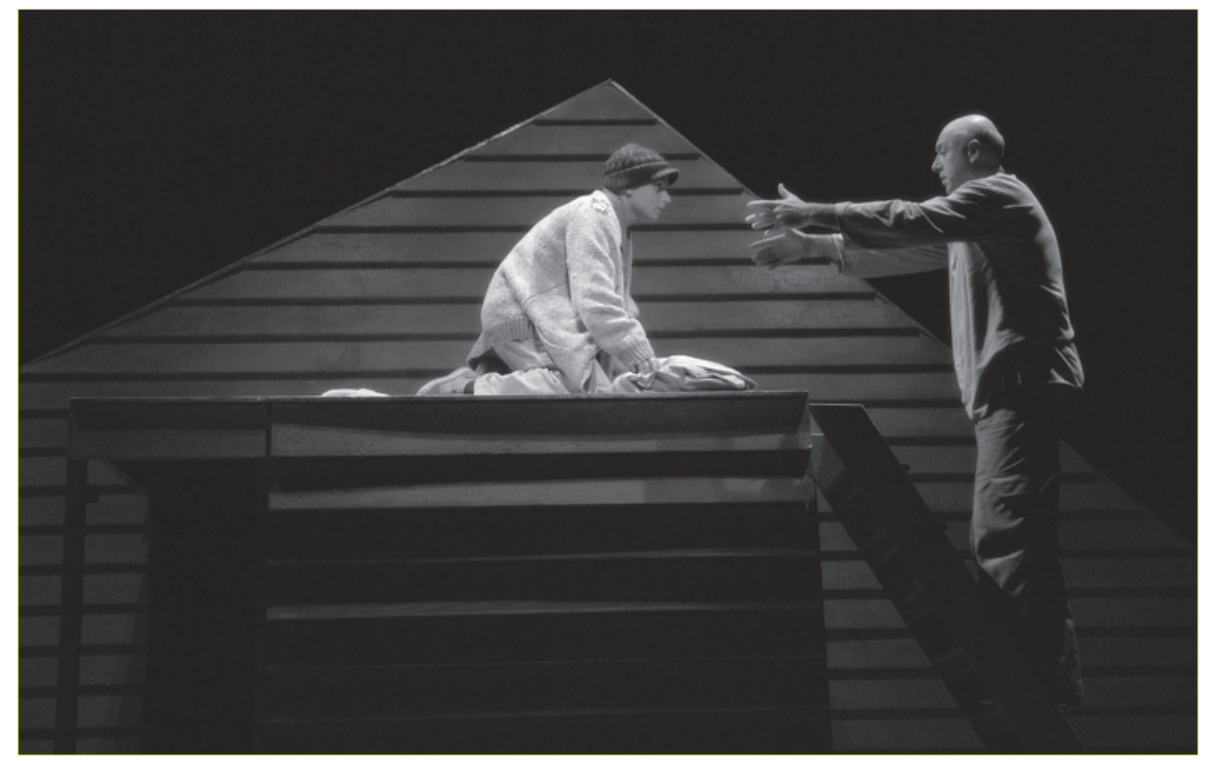

Terre océane de Daniel Danis. Mise en scène de Gill Champagne, coproduction du Thêâtre de Quat'Sous, du Théâtre du Trident, de Logomotive Théâtre (Elbeuf sur Seine, France) et de la Compagnie Daniel Danis, arts/sciences, au Théâtre d'Aujourd'hui (Montréal), 2007. Avec, de gauche à droite, Sébastien René (Gabriel) et François Clavier (Antoine).

Photo: Yanick MacDonald. 